\title{
LOW PROFILE MULTI-FREQUENCY DIPOLE ANTENNA ARRAY BASED ON PLANAR META-SURFACES
}

\author{
Elena Saenz ${ }^{(1)}$, Pekka Ikonen ${ }^{(2)}$, Ramon Gonzalo ${ }^{(1)}$, \\ Iñigo Ederra ${ }^{(1)}$, Sergei Tretyakov ${ }^{(2)}$ and Peter de Maagt ${ }^{(3)}$ \\ (1) Electrical and Electronic Engineering Department, Public University of Navarra, \\ Campus Arrosadia, E-31006, Pamplona, Navarra, Spain \\ Email: elena.saenz@unavarra.es,ramon@unavarra.es, inigo.edera@unavarra.es
}

\author{
(2) Radio Laboratory/SMARAD, Helsinki University of Technology, \\ P.O. Box 3000, FI-02015 TKK, Finland \\ Email:pekka.ikonen@tkk.fi,sergei.tretyakov@tkk.fi
}

(3) European Space Research and Technology Centre, ESTEC, P.O. Box 299, 2201 AG Noordwijk, The Netherlands

Email: Peter.de.Maagt@esa.int

\begin{abstract}
In this paper, the radiation performance of a low profile multi-frequency dipole antenna array with a planar meta-surface is presented. The meta-surface consists of two closely located parallel grids of short dipoles and one grid of continuous wires in between. By tuning a dipole to the pass band of the superstrate and due to the magnetic dipole moments induced in the unit cells, a uniform illumination is achieved and therefore an enhancement of the directivity. Placing a second meta-surface under the dipole with the stop-band tuned to the working frequency, the back radiation is reduced. By combining low and high resonant-frequency unit cells and tuning dipoles to these frequencies, a double-frequency array is formed.
\end{abstract}

\section{Introduction}

Nowadays, due to the new requirements, many applications working at different frequencies are integrated in a single device. In order to cover all these features, low profile multi-frequency antennas are required. Several structures based on metamaterials have emerged as a possible technology for the gain enhancement of low gain radiators. One of the most promising structures are superstrates having ultra-low refractive index [1]. For small antennas, meta-surfaces as superstrates with a transmission window at the operating frequency band have been proposed in [2] - [3].

In this paper, a multi-frequency dipole antenna array (MFAA) is proposed, based on the success of the single-frequency antenna presented in [3]. The fundamental radiation properties of the structure shown in Fig. 1 (a) are studied. The first and third grids consist of parallel dipoles while the second grid is formed by solid wires. The main idea of that design is to excite resonant oscillations in the superstrate inclusions (this way creating a wider radiating aperture), maintaining partial transparency of the structure. At the resonant frequency, a strong magnetic dipole moment is induced in the cells, enhancing the transmission of power. Compared to the conventional wire array / split-ring resonator configurations, a clear advantage of the proposed structure is a planar design that simplifies the manufacturing process.

\section{Unit cells}

The proposed meta-surface is based on the periodic repetition of the unite cell shown in Fig. 1 (b). This unit cell exhibits a pass band around the resonant frequency, due to the compensation of the currents between the different layers, and a stop band at higher frequencies. In order to create a MFAA, two unit cells with resonant frequencies around 10 and $12 \mathrm{GHz}$ are used, the low-resonant-frequency unit cell (LRF) and the high-resonant-frequency (HRF) unit cell.

As it will be explained in the last section, a third unit cell working at the stop band will be used to create a substrate in order to enhance the boresight radiation reducing at the same time the back radiation.

The $S$ parameters of the three unit cells are shown in Fig. 1 (c). It can be observed that the two cells that will be used to create the superstrate (LRF and HRF) have the pass band where the substrate unit 


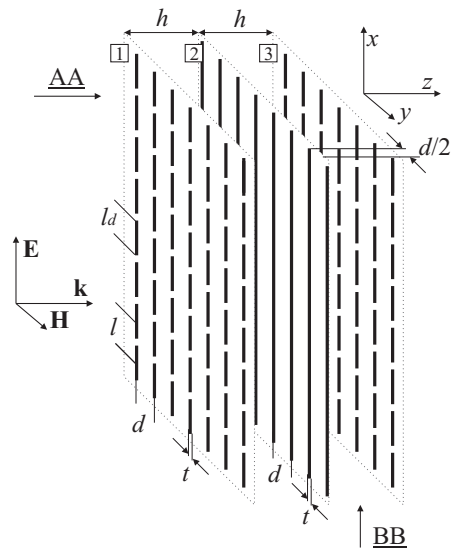

a)

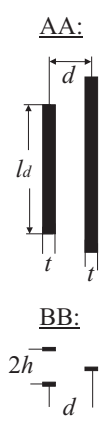

b)

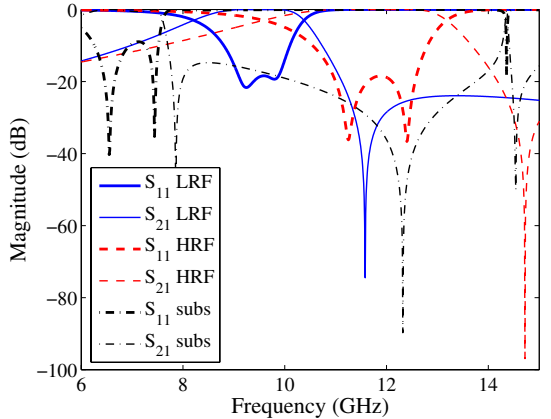

c)

Figure 1: (a) Proposed superstrate geometry. (b) Unit cell geometry. (c) $S$ parameters of the LRF, HRF and substrate unit cells.

cell has the stop band.

The dimensions of the unit cells are shown in Table 1 . In order to manufacture the three cells with the same material (RT-Duroid 5880, thickness $=780 \mu \mathrm{m}$ ), the thickness of the layers $h$ was fixed to $0.787 \mathrm{~mm}$ and the other dimensions were varied to get the desired resonant frequency and maximum bandwidth.

Table 1: Dimension ( $\mathrm{mm}$ ) of the unit cells.

\begin{tabular}{|c|c|c|c|c|c|c|}
\hline Cell & $l_{d}$ & $l$ & $t$ & $d$ & $h$ & $\varepsilon_{r}$ \\
\hline LRF & 9.17 & 10.37 & 0.9 & 1.6 & 0.787 & 2.2 \\
HRF & 7.36 & 8.56 & 0.7 & 1.6 & 0.787 & 2.2 \\
Subs & 13.6 & 16.32 & 0.8 & 3.2 & 0.787 & 2.2 \\
\hline
\end{tabular}

\section{Superstrate configuration}

As it has been explained, the LRF and HRF cells exhibit a pass band around 10 and $12 \mathrm{GHz}$, respectively. Combining both cells in two different regions of a meta-surface, and tuning two dipoles to each pass band, a MFAA can be designed. The geometry of the MFAA is shown in Fig. 2 (a).

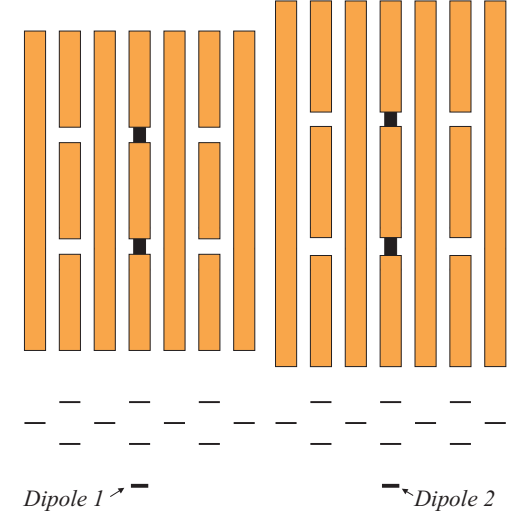

(a)

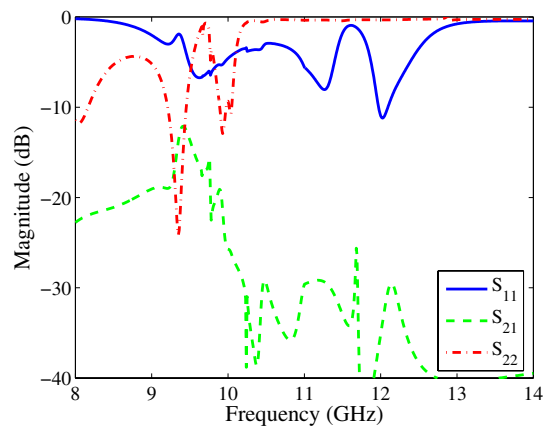

(b)

Figure 2: (a) Geometry of the MFAA with superstrate; top and transversal views. (b) $S$ parameters.

Dipole 1 is tuned to the high frequency HRF (12 GHz) and the dipole 2 to the low one LRF (10 GHz). The distance between the dipole and the superstrate is $1.574 \mathrm{~mm}$. So, the total thickness of the configuration (dipole + superstrate) is $3.148 \mathrm{~mm}$, i.e., $\lambda_{0} / 10$ at $10 \mathrm{GHz}$ or $\lambda_{0} / 8$ at $12 \mathrm{GHz}$. The physical area of the LRF region is $0.4 \lambda_{0}^{2}$ at $10 \mathrm{GHz}$ and the $\mathrm{HRF}$ ones $0.48 \lambda_{0}^{2}$ at $12 \mathrm{GHz}$. The distance between the dipoles 


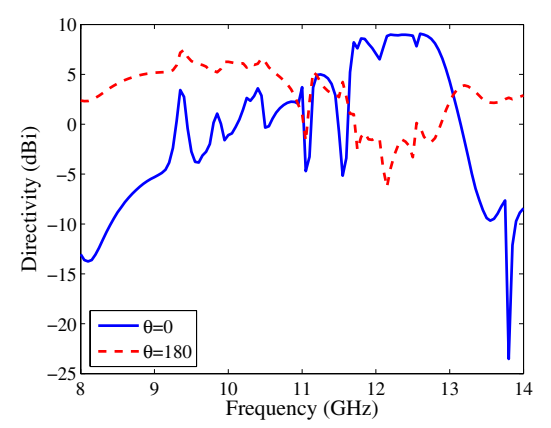

(a)

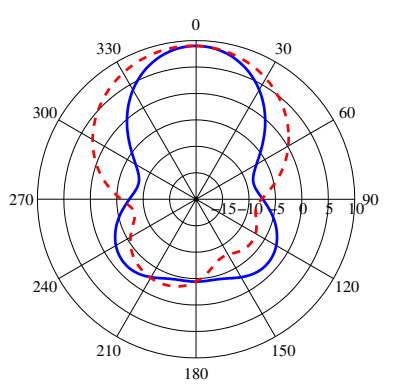

(b)

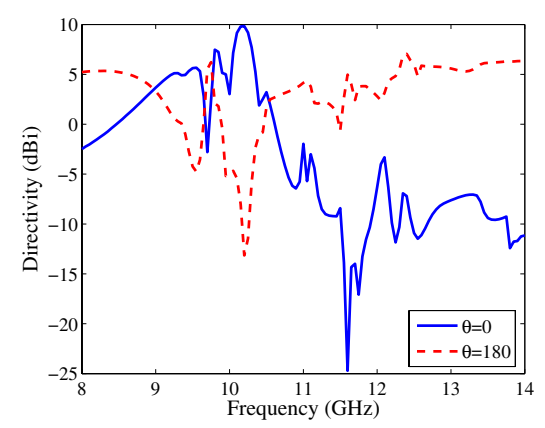

(c)

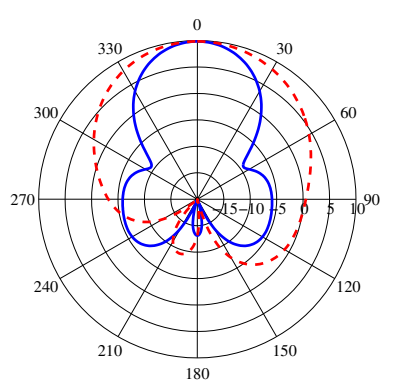

(d)

Figure 3: Directivity at $\theta=0^{\circ}$ and $\theta=180^{\circ}$. H-plane (continuous line) and E-plane (dashed line) radiation patterns. (a) (b) Dipole 1 is radiating $f_{r}=12.2 \mathrm{GHz}$. (c)(d) Dipole 2 is radiating $f_{r}=10.2 \mathrm{GHz}$.

is $11.2 \mathrm{~mm}, \lambda_{0} / 2.5$ approximately at the LRF. The $S$ parameters of the structure are shown in Fig. 2 (b), where a very low coupling between elements can be observed $\left(S_{21}<-20 \mathrm{~dB}\right.$ at the working frequencies).

The directivity and radiation patterns have been analyzed separately when dipole 1 was radiating (Fig. 3 (a) and (b)) and when dipole 2 was (Fig. 3 (c) and (d)). In both cases, an enhancement of the boresight radiation $\left(\theta=0^{\circ}\right.$ continuous line $)$ and reduction of the back radiation $\left(\theta=180^{\circ}\right.$ dashed line $)$ was observed.

Taking into account the directivity $D$ and the physical area $A_{p h y}$, the aperture efficiency $\eta_{a p}$ has been calculated as follows.

$$
D=\frac{4 \pi}{\lambda_{0}^{2}} \eta_{a p} A_{p h y}
$$

The maximum directivity achieved for the $\mathrm{LRF}$ and $\mathrm{HRF}$ regions was $9.84 \mathrm{dBi}$ at $10.2 \mathrm{GHz}$ and $9 \mathrm{dBi}$ at $12.2 \mathrm{GHz}$, which means an aperture efficiency of 1.9 and 1.4 respectively.

\section{Substrate-superstrate configuration}

As it is shown in Fig. 1 (c), the substrate cell exhibits a stop-band just where the LRF and HRF cells have the transmitting windows. In order to reduce the back radiation of the previous superstrate configuration, a substrate based on this substrate cell is placed above the radiating dipoles. Although the distance from the dipoles to the superstrate and substrate is very small, $0.787 \mathrm{~mm}$, since the currents induced in the passive dipoles are in phase with the radiating ones, the radiation efficiency is not lost. The total thickness of the configuration, taking into account the superstrate, the substrate and distance between them is $4.722 \mathrm{~mm}$, i.e., $\lambda_{0} / 6.5$ at the $\operatorname{LRF}(10 \mathrm{GHz})$ or $\lambda_{0} / 5.5$ at the HRF $(12 \mathrm{GHz})$. As in the previous case, the distance between active dipoles is $11.2 \mathrm{~mm}, \lambda_{0} / 2.5$ approximately at the LRF. The geometry of the MFAA with substrate and superstrate and the $S$ parameters are shown in Fig. 4 (a) and (b), respectively. $S_{11}$ and $S_{21}$ values around $-10 \mathrm{~dB}$ at the resonant frequencies have been obtained, with very low coupling between elements.

The $\mathrm{H}$ and E-plane radiation patterns have been calculated for both dipoles at its corresponding resonant frequency, $11.95 \mathrm{GHz}$ in the case of dipole 1 and $9.89 \mathrm{GHz}$ for dipole 2 (see Fig. 5). Comparing these 


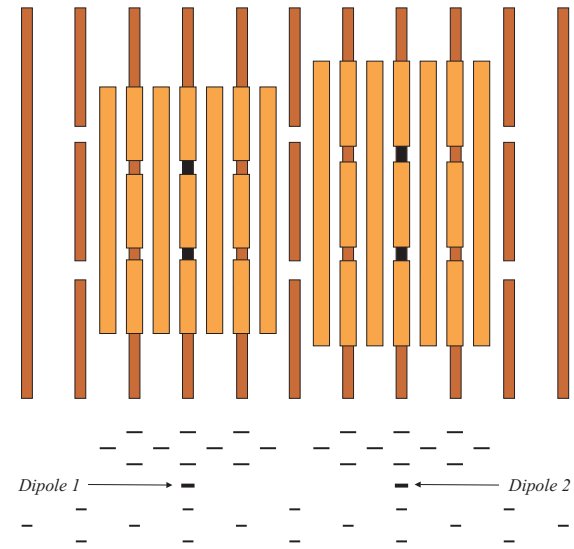

(a)

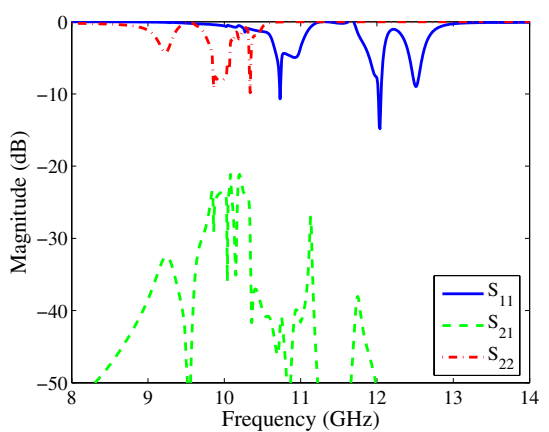

(b)

Figure 4: (a) Geometry of the MFAA with substrate and superstrate; top and transversal views. (b) $S$ parameters.

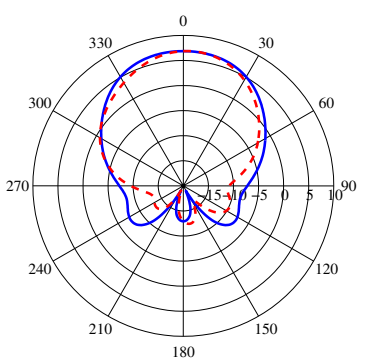

(a)

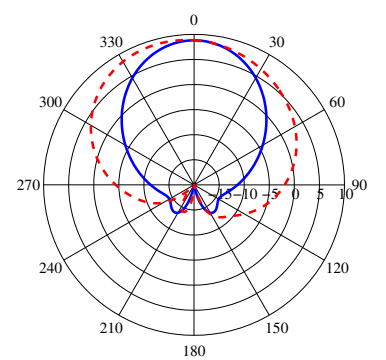

(c)

Figure 5: H-plane (continuous line) and E-plane (dashed line) radiation patterns. (a) Dipole 1 is radiating $f_{r}=11.95 \mathrm{GHz}$. (b) Dipole 2 is radiating $f_{r}=9.89 \mathrm{GHz}$.

radiation patterns with the previous case, i.e., in the absence of substrate, a reduction of more than 10 $\mathrm{dB}$ of the back radiation has been realized, obtaining a front-back radiation value close to $30 \mathrm{~dB}$.

\section{Conclusions}

In this paper, the radiation properties of a low-profile multi-frequency antenna array with meta-surfaces based on grids of dipoles and continuous wires have been analyzed. The low and high frequency regions of the superstrate were formed by two unit cells with pass-band at $10 \mathrm{GHz}$ and $12 \mathrm{GHz}$. By means of a third cell with the stop band from 8 to $13 \mathrm{GHz}$, a substrate has been designed in order to reduce the back radiation. By tuning two dipoles to the transmitting windows of the superstrate, and due to the induced magnetic dipole moments, high aperture efficiency is achieved, which produces an enhancement of the directivity as well as a reduction of the back radiation. Since the meta-surfaces are based on planar technology, the whole radiating system results very thin $\left(\lambda_{0} / 10\right.$ in the superstrate case, $\lambda_{0} / 6$ in the substrate-superstrate case) and simplifies the fabrication and testing process.

\section{References}

[1] S. Enoch, G. Tayeb, P. Sabouroux, N. Guerin, P. Vincent, "A metamaterial for directive emission," Phys. Rev. Lett., vol. 89, no. 21, pp. 3902, 2002.

[2] E. Saenz, R. Gonzalo, I. Ederra, P. de Maagt", "Application of left handed superstrates to improve radiation performances of dipole antennas," European Microwave Association, vol. 2, pp. 3-11, 2006.

[3] E. Saenz, R. Gonzalo, I. Ederra, P. de Maagt, "Design of a plannar meta-surface based on dipoles and wires for antenna applications," Proceed. EuCAP, 2006. 